tion mortels, et ordonner une enquête serrée dans le quartier, la ville, la commune ou eut lieu l'accident;

20 Réglementer l'industrie des fromages, en accord avec les municipalités et les services de Santé publique ;

$3^{\circ}$ Employer un lait pasteurisé ou faire bouillir convenablement le lait destiné à l'industrie du fromage ;

$4^{\circ}$ Distribuer, sous contrôle chimique, une présure aseptique ;

$5^{\circ}$ Conserver le surplus du fromage frais dans des frigidaires, au même titre que le beurre.

(Clinique médicale infantile de l'Hôpital des Enfants-Malades de Beyrouth.)

\title{
- ASPECTS SCIENTIFIQUES DE LA FABRICATION CONTINUE DU BEURRE (1)
}

\author{
par
}

\section{N. $\mathrm{KING}$}

Section des Recherches laitières. Organisation de Recherches seientifiques et industrielles du Commonwealth, Melbourne (Australie)

Quoiqu'il soit possible de baratter directement le lait, la fabrication pratique du beurre utilise une concentration en deux temps. Dans le premier, qui est l'écrémage, la teneur en matière grasse passe de 3 à $4 \%$ à environ 30 à $40 \%$; dans le deuxième, le barattage et le malaxage, elle augmente de nouveau jusqu'à environ $82 \%$. La préparation de la matière grasse de beurre (butteroil), produit contenant environ $99 \%$ de matière grasse, peut être considérée comme un troisième temps.

En même temps que s'est développée l'industrie beurrière s'est manifestée une tendance accrue à passer du travail intermittent au travail continu. Ce passage, dans le cas de la centrifugation de la crème, s'est produit au cours des dix dernières années du siècle écoulé. Les essais pour placer aussi le second temps de la fabrication du beurre sous le régime de la continuité ("extracteur» de beurre de Johansson, "séparateur " de beurre de de Laval et "radiateur" de beurre de Salenius) eurent lieu assitôt après l'introduction de l'écrémage centrifuge ; mais, malgré cela, n'eurent pas de résultats pratiques. Après un assez long intervalle de temps, ces essais furent repris pendant les trente récentes années du siècle actuel avec le résultat qu'en peu de temps un certain nombre de nouvelles méthodes de fabrication continue du beurre furent perfectionnées,

(1) Dairy Science Abstracts, 1952, 14-4, 226. 
dont certaines sont actuellement utilisées d'une façon assez importante dans l'industrie laitière.

Pendant le second temps de la concentration, la matière grasse et la phase aqueuse restante (plus l'air incorporé) sont recombinées dans le système physico-chimique compliqué qu'est le beurre. Les méthodes continues nouvellement imaginées ont montré qu'il existe différents procédés pour arriver à ce but, les caractéristiques spécifiques de ces méthodes laissant, cependant, leur empreinte sur la structure physique du beurre en résultant. En outre, les propriétés du beurre sont étroitement liées à sa microstructure (opinion qui est de plus en plus acceptée dans d'autres domaines, par exemple dans celui des matières plastiques). On peut done tenir pour valable la formule suivante :

\section{Méthode de fabrication $\rightarrow$ Structure du beurre $\rightarrow$ Propriétés du beurre.}

Les propriétés du produit obtenu sont un facteur décisif du succès commercial d'une méthode de fabrication. Ainsi donc, pour régler convenablement les caractéristiques de ces nouveaux types de beurre, ee qui est naturellement une exigence d'une importance pratique de premier ordre, il faut avoir une connaissance approfondie de la nature physico-chimique intime des phénomènes mis en œuvre. Le phénomène principal dans ces méthodes est l'inversion de phase. D'autres phénomènes importants sont la cristallisation de la matière grasse dans les globules et dans la matière grasse libre, et les modifications thixotropiques. En dehors des aspects pratiques, ces nouvelles méthodes de fabrication du beurre ont donc étendu nos connaissances des principes théoriques du barattage.

La position des méthodes continues modernes jusqu'en 1948 a été passée en revue et examinée par WIEChERs et DE GOEDE dans leur livre "Fabrication continue du beurre" (1950) (1). Au cours du XIIe Congrès International de la Laiterie à Stockholm (1949), la fabrication continue du beurre a été un des trente-six sujets mis en discussion (KING, 1949 b).

\section{Classification des méthodes}

Schulz (1947) a proposé un classement des méthodes et a aussi donné un plan pour l'évaluation et l'appréciation de différentes méthodes (Schulz et Schulz, 1948 ; Schulz, 1949). Dans ce plan, les avantages et les inconvénients des méthodes continues ont été comparés avec la méthode ordinaire de barattage.

(1) Note du Tradueteur. Voir : Le Lait, tome XXI, septembre-octobre 195I; n० 308 , p. $529 \cdot 530$. 
Dans l'étude actuelle, un système de classification quelque peu modifié est utilisé et est donné dans le tableau qui suit :

TABLEAU I

CLASSIFICATION DES METHODES MODERNES DE FABRICATION CONTINUE DU BEURRE

\begin{tabular}{|c|c|c|c|c|}
\hline \multicolumn{2}{|c|}{$\begin{array}{c}\text { Barattage et malaxage } \\
\text { accélérés }\end{array}$} & \multicolumn{3}{|c|}{ Ré-écrémage } \\
\hline $\begin{array}{c}\text { Barattage et } \\
\text { malaxage } \\
\text { continus }\end{array}$ & $\begin{array}{c}\text { Barattage } \\
\text { semi-continu } \\
\text { sous pression } \\
\text { de } \mathrm{CO}^{2}\end{array}$ & $\begin{array}{c}\text { Inversion de phase } \\
\text { par refroidissement } \\
\text { et traitement mé- } \\
\text { canique de la crè- } \\
\text { me réécrémée (état } \\
\text { de l'émulsion non } \\
\text { modifié) }\end{array}$ & $\begin{array}{l}\text { Rupture de l'émul- } \\
\text { sion de crème avant } \\
\text { et pendant le ré- } \\
\text { écrémage suivie } \\
\text { par l'émulsionne- } \\
\text { ment du plasma } \\
\text { dans la matière } \\
\text { grasse, et par un } \\
\text { malaxage supplé- } \\
\text { mentaire }\end{array}$ & $\begin{array}{l}\text { Rupture de l'émul- } \\
\text { sion de crème après } \\
\text { réécrémage suivie } \\
\text { par l'émulsionne- } \\
\text { ment du plasma } \\
\text { dans la matière } \\
\text { grasse }\end{array}$ \\
\hline $\begin{array}{l}\text { Méthode } \\
\text { Fritz }\end{array}$ & $\begin{array}{l}\text { Méthode } \\
\text { EL Senn }\end{array}$ & $\begin{array}{l}\text { Méthode Alfa : } \\
\text { suédoise, allemand. } \\
\text { Méthode New-Way }\end{array}$ & $\begin{array}{c}\text { Méthode Cherry- } \\
\text { Burell (Gold'n } \\
\text { Flow) }\end{array}$ & $\begin{array}{c}\text { Méthode Creamery } \\
\text { Package }\end{array}$ \\
\hline
\end{tabular}

\section{Fabrication ordinaire du beurre}

Comme les méthodes continues mettant en jeu un barattage et un malaxage accélérés ont beaucoup de points communs avec le barattage ordinaire, il semble convenable de donner un aperçu concis de la théorie de cette méthode. En outre, il est nécessaire d'avoir un certain modèle dans la comparaison des méthodes.

\section{Théorie du barattage et du malaxage}

D'après les exposés et les observations de Mlle Pockels (1902), de King (1931-1932) et de VAN Dam et Holwerda (1934), le barattage normal a lieu comme suit :

Par l'agitation de la crème, de l'air est incorporé dans le plasma de la crème sous forme de nombreuses petites bulles. Les globules de matière grasse arrivant à l'interface air-plasma de ces bulles y répandent une certaine proportion de leur matière grasse liquide. Une partie de cette matière grasse répandue reste liée aux globules comme une sorte de ceinture autour d'elles. Si les globules sont suffisamment rapprochés les uns des autres, cette ceinture les rassemble pour former des grumeaux. Ces grumeaux ne peuvent pas être brisés en simples globules par l'agitation et s'ils sont chauffés 
au-dessus du point de fusion de la matière grasse, un grumeau forme un seul gros globule de matière grasse. L'autre partie de la matière grasse dispersée existe sur la surface de la bulle à l'état de petits points de matière grasse avec une mince couche moléculaire entre eux. La mince couche moléculaire agit pour faire s'affaisser la mousse en produisant l'éclatement et l'effondrement des bulles d'air (protégées probablement auparavant par des protéines du lait). Par l'éclatement des bulles, les petits points do matière grasse sont dispersés dans une partie du plasma de la erème sous forme de très petits globules de matière grasse. Ceux-ci passent dans le babeurre, produisant les pertes de matière grasse pendant le barattage. Les fragments de couche moléculaire mince forment de plus petites particules de matière grasse, probablement de dimensions colloïdales. L'incorporation de l'air et la destruction des bulles est répétée à de nombreuses reprises, ce phénomène étant dynamique, avec pour résultat que de plus en plus de globules de matière grasse sont réunis aux grumeaux, qui, par les chocs, se forment en granules de beurre. Simultanément, a lieu un enrichissement de la matière grasse répartie très finement dans le babeurre.

Qu'ils soient lavés ou non lavés, salés ou non salés, les granules de beurre, lors du travail ultérieur se réunissent à la masse de beurre agglomérée. D'après KING $(1947, a)$, au fur et à mesure du malaxage, de plus en plus de globules de matière grasse sont écrasés et la proportion de matière grasse libre est ainsi continuellement augmentée. Toutefois, lorsqu'elle a atteint une certaine importance, la proportion de matière grasse globulaire ne présente plus que de très légères modifications ultérieures. En même temps, la phase aqueuse est de plus en plus subdivisée et l'đ humidité libre » disparaît. Une certaine proportion d'air, environ 3-4\% en volume, est incorporée, formant probablement de petites crevasses et fissures internes dans la masse du beurre et donnant au beurre un certain degré de friabilité. Si l'air est enlevé par le travail sous vide (BARNICOAT, 1947), la pâte du beurre devient serrée et dure (le beurre prélevé par la sonde à échantillonner étant souple) et ressemble au beurre. Alfa.

A un certain moment du barattage, par l'emploi d'une pression optimum sur les globules de matière grasse, les molécules de matières grasses à point de fusion le plus élevé sont orientées vers une couche périphérique biréfringente, cette couche donnant aux globules un certain degré de résistance contre la rupture (KING, 1950 b). Tous les globules de matière grasse dans le beurre terminé présentent cette couche. Ces globules peuvent être isolés par dilution soigneuse du beurre avec la fraction liquide de la matière grasse de beurre, 
puis ensuite comptés et mesurés (KING, $1947 a, b, d, f$, ; et BaUR, 1949).

\section{Structure physique du beurre ordinaire}

Le beurre représente une émulsion compliquée du type : "globules de matière grasse et gouttelettes de plasma dans la matière grasse libre " (KING, 1930). La matière grasse existe done dans le beurre sous deux formes différentes, comme globules de matière grasse ou matière grasse globulaire et comme matière grasse libre. Une partie de la matière grasse sous ces deux formes est à l'état cristallisé et un peu à l'état liquide. La dureté et la consistance du beurre dépendent done de la proportion et de la composition de ces deux formes de matière grasse (WodE, 1933 ; SAMutusson, 1937). L'incorporation d'air dans le beurre forme des crevasses internes et peut à un certain degré contribuer à la consistance du beurre (Schulz, 1948). D'après Mulder (1941) la 'phase grasse continue est interdispersée par un système de réseau d'interfaces aqueuses très minces, le beurre ayant ainsi deux phases continues.

Le diamètre moyen des globules de matière grasse dans le beurre est d'environ 3,5 à $4 \mu$. Ils sont sphériques, entourés d'une couche biréfringente, constituée par les molécules des matières grasses à point de fusion le plus élevé, orientée radialement par rapport à la surface du globule. Ce ne sont que des globules occasionnels qui contiennent de petites aiguilles de matière grasse. Il y a environ 18 à $28 \%$ de matière grasse globulaire dans le beurre ordinaire (MоHR et BAUR, 1949). La matière grasse libre ne contient ordinairement pas de cristaux de matière grasse visibles microscopiquement. Le beurre ayant le défaut dénommé "farineux " possède une certaine quantité de eristaux dans la matière grasse libre.

Les gouttelettes de la phase aqueuse ont un diamètre d'environ 1 à $30 \mu$ et on peut même trouver quelques gouttes plus grandes. Les gouttelettes sont généralement sphériques; elles ne contiennent pas de globules de matière grasse, même pas de petits, et n'ont jamais de couche biréfringente.

Dans un spécimen ordinaire, l'incorporation d'air n'est habituellement pas visible.

\section{Méthode Fritz}

Dans la méthode Fritz, la crème douce d'une teneur d'environ 45 à $50 \%$ de matière grasse pasteurisée et refroidie normalement, est soumise à une agitation vigoureuse par un rotor tournant dans un cylindre de barattage horizontal. Le babeurre s'écoule des granules de beurre dans une chambre inclinée, après quoi les 
granules passent sous pression à travers une série de plaques perforées et d'ailettes travaillantes à la partie supérieure de la chambre. Le beurre débouche d'une embouchure carrée, prêt à entrer dans une machine à mouler. Le cylindre de barattage et une partie de la section de la chambre sont munis d'une double enveloppe à circulation de saumure. Alors que dans la méthode habituelle, le barattage et le malaxage prennent à peu près une heure et demie, ils sont effectués ici en quelques minutes.

Les progrès de la méthode Fritz ont été décrits et discutés par Mennicke (1940 $a, b, 1942)$, Fritz, Müller et Mennicke (1941) et par Fritz $(1946,1949)$; une nouvelle construction de la machine (construction "Westfalia") et son fonctionnement dans l'agencement de l'installation sont décrits par WILSMANN et FELTENS $(1947,1950)$.

\section{Théorie du procédé Fritz.}

KING (1950 a) donne l'explication suivante des opérations dans la machine Fritz:

Alors que dans le barattage ordinaire, les globules de matière grasse sont rassemblés sur les surfaces d'un grand nombre de minuscules bulles d'air dispersées dans le plasma de la erème, les globules de matière grasse dans la machine Fritz sont rassemblés sur le grand interface air-crème constamment changeant de la mince couche de crème se déplaçant à grande vitesse sur un parcours spiralé de l'entrée à la sortie du cylindre de barattage. Toute la crème est étirée ou déformée (O\$W ALD, 1931) en une couche mobile très mince, dont une face est tournée vers la surface métallique refroidie et l'autre vers l'air. Une certaine proportion des globules peut, bien entendu, être aussi rassemblée à l'aide des petites bulles d'air incorporées dans la crème par l'agitation.

Aussitôt que la crème entrée dans le cylindre est étalée en couche mince un grand nombre de globules de matière grasse s'établissent sur cette surface. Par suite du courant turbulent de la couche de crème de plus en plus de globules sont précipités de l'intérieur de la couche à la surface. Exactement comme dans les opérations du barattage ordinaire du beurre, une partie de la matière grasse liquide est projetée hors des globules de la surface de la crème formant, d'une part, la ceinture autour des globules et, d'autre part, les petits points de matière grasse et la mince couche moléculaire. Alors que la ceinture dans son rôle de substance de masticage des globules va d'abord dans les grumeaux, puis dans les granules de beurre puis ultérieurement dans le beurre, les petits points et la mince couche moléculaire sont dispersés sous une forme très menue dans le babeurre, produisant ainsi la perte de matière 
grasse qui est plus grande dans le babeurre Fritz que dans le babeurre ordinaire. La teneur en matière grasse du premier peut facilement atteindre une valeur aussi élevée que $1 \%$.

Wilsmann et Felten (1950) font valoir l'importance de la couche biréfringente des globules dans la formation du beurre Fritz. Par l'action du rotor, quelques-uns des globules sont broyés, fournissant des substances pour enrober les globules laissés en arrière. Si la solidification des globules a été trop loin, aucune matière grasse liquide n'est libérée et, ni grumeaux, ni granules ne sont formés.

Le pourcentage de la matière grasse globulaire et la forme des globules sont sous l'influence : $1^{\circ}$ du rendement de la machine et $2^{\circ}$ de la température de la crème (KING, $1947 e$; KING et FrITz, 1948). Avee un rendement faible, les globules sont soumis pendant une plus longue durée au traitement mécanique et plus de globules sont broyés, la proportion de matière grasse libre étant ainsi augmentée. D'autre part, avec un rendement plus élevé, le pourcentage de matière grasse libre est plus faible. Plus la température de la crème est élevée, moins il existe de matière grasse solide dans les globules qui, par conséquent, sont moins résistants à l'action mécanique du rotor, et plus faible sera la teneur du beurre en matière grasse globulaire.

L'aspect microscopique des globules de matière grasse est aussi sous l'influence de ces deux facteurs. Avec un rendement élevé et avec des températures basses, les globules sont régulièrement sphériques et entourés par une couche biréfringente bien formée. Avec un rendement plus faible et avec des températures élevées, les globules deviennent déformés.

\section{Structure et propriétés du beurre Fritz.}

Le pourcentage de matière grasse globulaire dans le beurre Fritz est indiqué par MoHR et BAUR (1949) comme étant de 12 à $17 \%$. De même que dans le beurre ordinaire, on ne voit pas de cristaux de matière grasse dans la matière grasse libre du beurre Fritz.

D'après Wilsmann et Feltens (1950), la teneur en humidité du beurre Fritz dépend de la quantité de matière grasse libre qu'il contient, la teneur en humidité augmentant avec le pourcentage de matière grasse libre. Il en résulte que toutes les mesures augmentant la quantité de matière grasse libre sont susceptibles d'augmenter la teneur en humidité, e'est-à-dire : $1^{0}$ une plus grande vitesse du rotor; $2^{\circ}$ la forme des ailes batteuses ; $3^{\circ}$ des températures plus élevées ou un traitement préliminaire de la crème produisant des couches biréfringentes plus minces et $4^{\circ}$ une teneur plus 
élevée de la crème en matière grasse. Dobinsky $\left(\begin{array}{lll}1950 & b\end{array}\right)$ insiste aussi sur le vieillissement de la crème associé au phénomène d'agglomération dans les globules de matière grasse.

En dépit de la courte durée du malaxage, le beurre Fritz est malaxé très efficacement. KING et Fritz (1948) soutiennent que le degré de dispersion de la phase aqueuse dans le beurre Fritz est plus élevé que dans le beurre ordinaire. Les récentes mesures de dimensions effectuées par Hoyos (1951) confirment cette constatation, les gouttelettes ayant un diamètre au-dessus de $15 \mu$ étant très rares. Hoyos insiste aussi sur l'importance qu'a la dimension des gouttelettes infectées par des bactéries sur la qualité de conservation $d u$ beurre. Un beurre dont les grandes gouttelettes sont infectées se conserve mieux que quand les plus petites gouttelettes le sont.

Pendant le barattage continu, il est de la plus grande importance de suivre constamment la teneur en humidité afin d'obtenir un rendement maximum. DoBinsky $(1950 a)$ a procédé à un essai pour appliquer la mesure de la constante diélectrique dans le cas de la fabrication du beurre par la méthode Fritz. La constante diélectrique dépendait : $1^{\circ}$ de la teneur du beurre en air ; $2^{\circ}$ de la dimension et du nombre des gouttelettes d'eau et $3^{\circ}$ mais à un degré moindre de la température et du phénomène de cristallisation dans la matière grasse.

$\mathrm{Si}$ on étudie la théorie des méthodes continues, la question de l'équilibre de la lécithine peut aussi être intéressante comme index de la conduite de la membrane du globule de matière grasse pendant le barattage (Fritz, 1944). Moнr (1950 b) a trouvé que la teneur en phosphatide du beurre Fritz était plus élevée que celle du beurre ordinaire et inférieure à celle du beurre Alfa. La teneur en phosphatide du babeurre Fritz était plus élevée que celle du babeurre ordinaire. Cette constatation est, toutefois, en rapport avec la plus forte teneur en matière grasse du babeurre Fritz, comme l'ont indiqué Kieferle, Frichtner et Pirner (1950), qui ont trouvé que la teneur en lécithine par gramme de matière grasse était encore plus élevée dans le beurre ordinaire.

Quoique la teneur en air du beurre Fritz soit sensiblement plus élevée que celle du beurre ordinaire, étant d'environ $7 \%$ en volume contre environ 3\% (Schulz, 1938), les bulles d'air ne sont généralement pas visibles dans un spécimen microscopique.

C'est probablement en raison de sa plus forte teneur en air que le beurre Fritz a des tendances à devenir friable (Fritz, 1949). L'incorporation de l'air donne manifestement naissance à un certain nombre de crevasses et de fissures internes dans la masse du beurre. L'effet semblable de crevasses internes dans les métaux, 
le verre et les matières plastiques a été étudié par SMekaL (1936, 1949). La tendance du beurre Fritz après conservation au froid (Pirner, 1946 ; Stöfrler, 1950), les petites gouttelettes d'eau se réunissant en gouttelettes plus grandes en produisant de l'humidité libre, tout cela est probablement relié à l'existence de ces crevasses internes. Le beurre Fritz doit done être mis en morceaux très rapidement après fabrication.

\section{Méthode Senn}

La crème, qu'elle soit douce ou acide, est barattée d'une manière discontinue par fournées successives dans une petite baratte avec agitateurs verticaux sous une pression de 4 à 5 atmosphères d'acide carbonique. Après drainage et soutirage du babeurre, les granules de beurre sont lavées puis pressées ensemble (comprimées), un malaxage réel du beurre étant, d'après SENN, indésirable pour la pâte du beurre.

La méthode est basée sur les théories de WIEgner et Gessner et les essais pratiques de Feremutsch (Koestlen, 1934). D'après le précédent, le barattage sous pression d'acide carbonique peut être considéré comme un phénomène de coagulation dans lequel les globules de matière grasse stabilisés par hydratation et par charge électrique sont déstabilisés : $1^{\circ}$ par l'action de dégagement de l'acide carbonique en solution aqueuse; $2^{\circ}$ par un déplacement partiel de la substance protectrice de la surface des globules de matière grasse vers la surface des bulles d'acide carbonique et $3^{\circ}$ par la violente agitation des rotors.

Il n'existe que peu de renseignements sur ce procédé dans la littérature laitière (St üssi, 1946, 1948). Il a été indiqué que le beurre Senn a une tendance à l'émiettement et au défaut d'" humidité libre ", dûs probablement au malaxage insuffisant pendant le stade de la "compression ". De récents modèles de la machine Senn sont compris pour un travail plus complet du beurre (WILEy et Loftus Hill, 1951).

\section{Mléthode Alfa}

D'après Stigen, Bergeöf et Hansson (1949), il faut faire une distinction entre la méthode suédoise Alfa et la méthode allemande Alfa (Alfa-Butterung). La première a trait à un beurre salé et aromatisé avec une teneur en humidité n'excédant pas $16 \%$ tandis que l'autre fut créée à l'origine pour l'obtention d'un beurre non salé de crème douce contenant jusqu'à $20 \%$ d'humidité.

Les phases principales de la méthode Alfa sont le réécrémage de la crème primaire pour obtenir une crème à $80 \%$ et le refroidis- 
sement et le malaxage de ce produit pour obtenir le beurre Alfa. Ces opérations sont exécutées dans une sorte d'écrémeuse quelque peu modifiée et hermétiquement étanche, le "concentrateur " "et dans un appareil combiné de malaxage et de refroidissement, le "transmutateur" qui consiste en trois cylindres à double enveloppe refroidissante reliés en série et munis de rotors ayant des nervures en spirale à leur surface. Le transmutateur ressemble à un refroidisseur spécial, le "votator" utilisé dans l'industrie des huiles et des graisses. Une nouvelle construction allemande du transmutateur (BERGERDoRfer ErsenwERKe, 1950) consiste en une batterie de plaques circulaires avec espaces alternants pour la crème ou le beurre et pour la saumure réfrigérante, les premiers étant munis de grattoirs. Une telle construction convient au démontage rapide pour le nettoyage. Récemment, une pompe centrifuge a été ajoutée et est utilisée après le transmutateur dans le but d'améliorer la consistance du beurre, en particulier pour éviter le défaut dit de "laminage". Cette pompe donne au beurre Alfa un traitement mécanique supplémentaire.

Une caractéristique particulière de l'installation suédoise est l'appareil de dosage pour le sel, le mélange aromatique et le colorant consistant en une cuve de mélange et une table de dosage.

\section{Second écrémage.}

Lors du second écrémage, les globules de matière grasse sont amenés en contact tellement étroit qu'ils deviennent déformés (Mohr et Wellm, 1948 a). Cette déformation mutuelle des globules de matière grasse est considérée par Moнr et Welcm comme étant le premier pas de la formation du beurre. La forme originale de l'émulsion de la crème est, toutefois, encore conservée aussi longtemps que la température est au-dessus de $20^{\circ} \mathrm{C}$, c'est-à-dire que la crème à $80 \%$ peut être diluée avee du lait écrémé chaud en conservant le degré primitif de dispersion de la matière grasse (MoHR, 1946). Il semble, toutefois, qu'il se produise certaines modifications dans la membrane du globule de matière grasse. D'après Perlmann (1935), la teneur en phospholipide de la crème augmente uniformément avec la teneur en matière grasse jusqu'à environ 55 à $58 \%$, après quoi elle diminue si la proportion de matière grasse augmente eneore. Il semble donc possible qu'il se produise une usure de la substance de la membrane. Josephson et DAHLE (1936) indiquent aussi qu'il se produit une usure mécanique des substances de la membrane quand on écrème à une teneur au-dessus de $65 \%$, une telle crème produisant une séparation de la matière grasse dans les "mixes» (mélanges) pour crème glacée. JAck et DAHLE (1937). 
notent une diminution rapide du phosphore lipidique des globules de matière grasse associée à une montée brusque de la mobilité électrophorétique à environ 60 à $65 \%$ de matière grasse. D'après MoHr et Weldm $\left(1948\right.$ b), la viscosité de la crème mesurée à $40^{\circ}$ et à $60^{\circ}$ augmente rapidement à $65-70 \%$ de matière grasse. Cette usure de la substance de la membrane peut n'être que partielle, puisque la crème à $80 \%$ conserve son caractère d'émulsion graissedans-eau au-dessus de $20^{\circ}$.

Le lait écrémé provenant du concentrateur contient plus de matière grasse que le lait écrémé primitif $(0,04$ à $0,05 \%$ par extraction à l'éther); mais moins de matière grasse que le babeurre ordinaire $(0,5$ à $0,9 \%)$. D'après MuLDER et ses collègues (1949), la teneur en matière grasse du second lait écrémé est habituellement inférieure à $0,07 \%$ (par la méthode Gerber), ne s'élevant à $0,1 \%$ que dans quelques cas. LoFtus-HILls, BALLARD et Wilkinson (1950) indiquent $0,1 \%$ (par extraction à l'éther) comme étant la proportion la plus élevée pour le lait écrémé provenant de crème pasteurisée dans un appareil à plaques. McDowall et McWhirTer (1948) ont, toutefois, trouvé des proportions aussi élevées que $0,6 \%$ pour le lait pasteurisé provenant de crème pasteurisée instantanément.

La teneur en matière grasse du second lait écrémé est influencée par le pré-traitement de la crème. Elle est augmentée par l'agitation. pandant le transport et par la vacréation de la crème primitive (McDowall et MoWhirter, 1948 ; Loftus Hills et Coll., 1950), une légère vacréation l'augmentant jusqu'à $0,38 \%$ et une vacréation poussée jusqu'à $0,49 \%$.

L'utilisation du second lait écrémé acidifié comme succédané du babeurre a été étudié par Moнr et HäsING (1949) et par MонR. et MACK (1944).

(A suivre)

\section{REVUE}

\section{PRODUCTION DE LAIT ET DÉRIVÉS EN EUROPE ET DANS LE MONDE (1)}

La production de lait de vache, qui était en 1949 sensiblement voisine de celle d'avant-guerre, a dépassé celle-ci en 1950, et a marqué une autre augmentation en 1951.

Nous indiquons ci-dessous les données de la production mondiale de lait de vache :

(1) Extrait de $1 l$ Mando del Latte, 1952, 7, 391. 